\title{
Guiding Principles for Cancer Surgery during the COVID-19 Pandemic
}

\author{
S. V. S. Deo ${ }^{1}$. Sunil Kumar ${ }^{1}$ - Naveen Kumar ${ }^{1}$. Jyoutishman Saikia ${ }^{1} \cdot$ Sandeep Bhoriwal ${ }^{1} \cdot$ Sushma Bhatnagar ${ }^{2}$. \\ Atul Sharma ${ }^{3}$
}

Published online: 6 May 2020

(C) Indian Association of Surgical Oncology 2020

\section{Introduction}

COVID-19 pandemic had an unprecedented adverse impact on health care services globally. This pandemic had started in the Wuhan city of China where the first case was reported on 31st December 2019 [1, 2] and on 11 th March 2020 the World health organization (WHO) had declared it as a global pandemic and as a public health emergency of international concern [3]. Within a short span of 3 months a significant proportion of different geographic regions of the world are facing the impact of COVID-19 pandemic [4-6]. Government of India has imposed a nationwide lockdown from 24th March 2020 to contain the spread of coronavirus infection. The pandemic has caused major disruption of health care services both in high income and low and middle income countries with limited resources. The challenges faced by health care sector include, caring for critical COVID-19 patients in hospitals resulting in massive diversion of critical hospital resources, caring for non COVID-19 patient population with medical and surgical emergencies and last but not the least - protecting health care providers (HCP) and implement new infection control protocols. In most of the hospitals worldwide, surgeons are operating only on patients with life threatening emergencies and postponing majority of elective surgical cases. The challenges surgical community facing include screening for COVID status, protection of HCP, judicious use of limited personal protective equipment (PPE) and other hospital resources.

S. V. S. Deo

svsdeo@yahoo.co.in

1 Department of Surgical oncology, BRA-IRCH, All India Institute of Medical Sciences, New Delhi 110029, India

2 Department of Onco-Anasthesia, All India Institute of Medical Sciences, New Delhi, India

3 Department of Medical oncology, All India Institute of Medical Sciences, New Delhi, India
Major factors that will guide surgical practice in the current scenario are stage of COVID-19 pandemic in a particular country / region and availability of health care resources. Most of the countries are now reaching the stage of community spread of COVID-19 infection with a huge number of potential asymptomatic carriers and significant number of critical COVID-19 patients.

Based on the COVID-19 status of the region/hospital and availability of health care resources American College of Surgeons (ACS) has proposed 3 different phases that a health care setup can encounter. Phase 1 - Semi-urgent settings (Preparation phase): The disease is not in the rapid escalation phase and institutions have adequate resources such as hospital and ICU beds, ventilators and manpower to cater the services. Phase 2 - Urgent settings: Limited availability of resources due to increased number of COVID-19 patients. Phase 3- Hospitals are over burdened with COVID-19 patients and non-availability of health care facilities like operating rooms, beds, ICU and ventilators.

The challenges faced by surgeons treating cancer are unique, because most of the cancer surgeries are elective but cannot be delayed beyond a certain point of time due to biology of the disease and adverse impact on survival if surgery is delayed. Due to the protracted nature of COVID -19 pandemic surgical oncologists world over are facing ethical and moral dilemmas in day to day practice while taking decisions regarding cancer surgery. In order to overcome these challenges a number of scientific societies and organizations have recommended triaging of surgical patients and proposed guidelines for handling patients waiting for cancer surgeries. These organizations include American college of surgeons (ACS), society of surgical oncology (SSO), European society of surgical oncology (ESSO), National Comprehensive Cancer Network (NCCN), Irish head and neck society, British Association of Surgical Oncology (BASO) and British gynecological cancer society (BGCS) [7-12].

An attempt has been made in this article to summarize various recommendations and propose certain guiding principles which will help the surgeons treating cancer in making 
critical surgical decisions. These guiding principles are not based on any high level of clinical evidence due to the unprecedented nature of COVID-19 pandemic and treating teams should make individualized treatment decisions which are shared and multidisciplinary in nature depending on the local circumstances and status of the patient.

\section{Cancer Surgery - Challenges during COVID-19 Pandemic}

1. Apart from oncological emergencies, majority of cancer treatments are planned and elective in nature. However guidelines recommend that elective cancer surgeries should be given priority and should be performed in a time bound fashion due to the biology of the disease and impact on survival if treatment is delayed beyond a certain point of time.

2. Current management approach to cancer is multidisciplinary in nature and a significant proportion of patients receive pre or post-operative radiotherapy or systemic therapy(chemotherapy, targeted therapy or hormonal therapy) based on site, stage and histopathology.

3. In general cancer treatments take relatively long time (few months) to complete and involve multiple visits and admissions to hospital.

4. The field of cancer involves a diverse spectrum of diseases and clinical presentations with varied clinical trajectories.

5. Based on clinical presentation - Cancer patients can be grouped as patients presenting with oncological emergencies, patients presenting with early or locally advanced cancers which are potentially curable and patients presenting with advanced or metastatic disease suitable for palliation only.

6. Based on status of treatment cancer patients can be grouped in to - new patients presenting for workup and diagnosis, patients who have completed diagnosis \& staging and waiting for initiation of treatment, patients midway through the treatment process and patients coming for post treatment follow-up.

7. Majority of cancer patients are immune compromised and relatively higher fatalities have been reported in cancer patients infected with COVID [13-15].

\section{Proposed Guiding Principles for Cancer Surgeries during COVID Pandemic}

1. The key determinants of decision making for cancer surgery during covid pandemic are - status of covid pandemic, availability of resources, patient and tumor related factors.
2. The basic tenets of cancer care - Multi disciplinary treatment approach should be followed using virtual technologies. Involve medical oncology, radiation oncology and palliative care for shared decision making.

3. Involve the patient and family in the decision making and clearly document the shared management decision in the file.

4. Minimize hospital visits of new patients and advise basic necessary diagnostic investigations only.

5. Advise cancer patients who have completed treatments and are disease free to stay at home. Tele consultations can be offered to these patients.

6. Any treatment planning should be made in the context of current and emerging covid situation, facilitating completion of oncologically appropriate treatment protocols in near future.

7. Operate on patients presenting with onco- surgical emergencies with all precautions as recommended for any surgical emergency during covid pandemic.

8. Decisions regarding elective cancer surgeries should be individualized based on type, stage, biology, availability of non-surgical treatment options and status of resources in the treating center.

9. Whenever feasible offer non-surgical treatment options in consultation with medical and radiation oncology (eg.Tamoxifen for hormone receptor positive breast cancer, pre-operative chemo/radiation for rectal or esophageal cancers) to contain or down stage the disease and subsequently plan elective surgery.

10. Offer surgery to patients, when non-surgical treatment options are not available or if the treating team feels that delay of surgery threatens patient's survival chances.

11. Postpone elective cancer surgery in patients with less aggressive and slowly growing cancers (differentiated thyroid cancer, prostate cancer, low grade soft tissue tumors, DCIS, basal cell carcinoma etc.).

12. Avoid aerosol generating procedures whenever feasible including endoscopies and laparoscopies.

13. Involve the anesthesia and critical care teams for surgical, critical care and post op recovery planning.

\section{Key Points}

1. All recommendations and safety precautions pertaining to patient screening, preparation of operation theatre, PPE, Intubation protocols, critical care protocols, human resource management, biomedical waste disposal and specimen handling should be strictly followed as described for any emergency or elective cancer surgery during COVID pandemic $[16,17]$. 
2. These guiding principles are not based on high level evidence and can be considered as advisories to overcome the challenges posed by unprecedented COVID-19 pandemic affecting health care sector. Due to rapidly evolving nature of the pandemic these guidelines should not be considered as standard of care in the long term. Due to anticipated breakthroughs and advances related to understanding of COVID-19 management further modifications to the guidelines related to cancer surgery field are anticipated and surgical oncologists are recommended to access resources related to COVID-19 and cancer management on a regular basis for updates.
Proposed Organ Specific Guiding Principles

for Cancer Surgery Decision Making during the COVID-19 Pandemic for Common Cancers

(ACS- American College of Surgeons, SSO-Society of Surgical Oncology, NCCN - National Comprehensive Cancer Network, BASO- British Association of Surgical Oncology, AHNS- American Head \& Neck Society, IASLC - International Society for study of lung cancer)

\section{Breast Cancer}

\begin{tabular}{|c|c|c|c|c|}
\hline SL & $\begin{array}{l}\text { No } \\
\text { Suggested surgical } \\
\text { options }\end{array}$ & Subtypes & $\begin{array}{l}\text { Recommendations by (ACS, } \\
\text { SSO, NCCN) }\end{array}$ & $\begin{array}{l}\text { Suggested non-surgical } \\
\text { options }\end{array}$ \\
\hline 1. & $\begin{array}{l}\text { Benign/premalignant } \\
\text { lesions }\end{array}$ & Defer surgery for 3 months & & Defer surgery for 3 months \\
\hline 2 & $\begin{array}{l}\text { ER +ve DCIS } \\
\text { Premenopausal }\end{array}$ & $\begin{array}{l}\text { Tamoxifen versus aromatase inhibitor (at the discretion of } \\
\text { medical oncology) for 3-5 months. }\end{array}$ & $\begin{array}{l}\text { Tamoxifen/Aromatase } \\
\text { Inhibitors }\end{array}$ & Defer Surgery for 3 months \\
\hline 3 & ER-ve DCIS & $\begin{array}{l}\text { Low volume disease \& low clinical suspicion of invasion } \\
\text { - Defer surgery \& reassessment } \\
\text { Large volume disease - Reassessment } 4 \text { weekly for } \\
\text { progression, if progressed then plan for Surgery }\end{array}$ & & $\begin{array}{l}\text { Defer surgery \& reassessment } \\
\text { 4weekly } \\
\text { Reassessment } 4 \text { weekly for } \\
\text { progression, if progressed } \\
\text { then plan for Surgery }\end{array}$ \\
\hline 4 & Her+ or TNBC & $\begin{array}{l}\geq \mathrm{T} 2 \text { and any } \mathrm{N}-\mathrm{NACT} \\
\mathrm{T} 1 \mathrm{~N} 0 \text { - Can consider for surgery, Else NACT (as per local } \\
\quad \text { resources) }\end{array}$ & $\begin{array}{l}\text { NACT\& Reassess } \\
\text { Complete NACT if Stable } \\
\quad \text { disease, PR or CR }\end{array}$ & $\begin{array}{l}\text { Progression on or after } \\
\text { chemotherapy - Consider } \\
\text { for surgery }\end{array}$ \\
\hline 5 & $\mathrm{ER}+/ \mathrm{PR}+$ & $\begin{array}{l}\text { All Stages - Consider endocrine therapy for at least 3-5 } \\
\text { months (Tamoxifen/AI) } \\
\text { Reassessment } 4 \text { weekly } \\
\text { Can also start chemotherapy (If indicated) }\end{array}$ & $\begin{array}{l}\text { All stages- Tamoxifen/AI and } \\
\text { response assessment } \\
\text { Continue HT till progression }\end{array}$ & $\begin{array}{l}\text { Surgery only if progression } \\
\text { during HT }\end{array}$ \\
\hline 6 & $\begin{array}{l}\text { Post-neoadjuvant } \\
\text { chemotherapy }\end{array}$ & $\begin{array}{l}\text { Consider endocrine therapy (if PR/CR and ER+) and delay } \\
\text { surgery versus surgery in } 4-8 \text { weeks. Can add } \\
\text { anti-HER2 therapy along with endocrine therapy if } \\
\text { HER2+ } \\
\text { If TNBCdelay surgery for } \mathbf{4 - 8} \text { weeks }\end{array}$ & $\begin{array}{l}\text { Consider HT (for luminal } \\
\text { subtypes) and Anti - } \\
\text { HER2 therapy for HER2+ }\end{array}$ & $\begin{array}{l}\text { Delay surgery as long as } \\
\text { possible }\end{array}$ \\
\hline 7 & $\begin{array}{l}\text { Special cases - } \\
\text { Malignant } \\
\text { phylloides, } \\
\text { Angiosarcoma }\end{array}$ & Consider for surgery & & Consider for surgery \\
\hline
\end{tabular}

$C R$ complete response, $P R$ partial response, $S D$ stable disease, $H T$ hormonal therapy, $A I$ aromatase inhibitors, $N A C T$ neo-adjuvant chemotherapy 


\section{Colo-Rectal Cancer}

$\begin{array}{lll}\text { SL Subtypes } & \text { Recommendations by - ACS, SSO. } \\ \text { No } & \begin{array}{l}\text { Suggested non-surgical } \\ \text { options }\end{array} & \text { Suggested surgical options }\end{array}$

\section{SL}

1. Benign/premalignant lesions (polyps)

2 Early stage colon cancer

3 Locally advanced colon and Metastatic colon Cancer

$4 \quad$ Rectal cancer (all stages)

5 Emergency indications (Obstruction/perforation/bleeding)
Defer surgery

Defer surgery

Neoadjuvant therapy

Neoadjuvant CT+ RT (Prefer short course radiotherapy over long course radiotherapy)

Delay surgery for 12-16 weeks post neoadjuvant therapy

Emergency surgery (Diversion stoma or resection of primary depending on intraoperative findings and hospital resources)
Defer surgery/consider surgery if progression

Defer surgery/consider surgery if progression

Neoadjuvant therapy/ Defer surgery until progression or Oral capacetabine only emergency indications

Neoadjuvant therapy/ Defer surgery until progression or Oral capacetabine emergency indications

Pst neoadjuvant treatm ent - can consider oral capecitabine

Emergency surgery (Diversion stoma or resection of primary depending on intraoperative findings)

\section{Gastro - Esophageal Cancers}

\begin{tabular}{|c|c|c|c|c|}
\hline $\begin{array}{l}\text { SL } \\
\text { No }\end{array}$ & Subtypes & Recommendations by - ACS, SSO. & Suggested non-surgical options & Suggested surgical options \\
\hline 1. & $\begin{array}{l}\text { Very early \& } \\
\text { superficial screen } \\
\text { detected cancers. }\end{array}$ & Prefer endoscopic procedures & & Defer Surgery \\
\hline 2 & $\begin{array}{l}\text { Early stage operable } \\
\text { Cancers }\end{array}$ & Surgery & $\begin{array}{l}\text { Esophageal cancer -NACT/ NACT+RT } \\
\text { Gastric cancer - NACT }\end{array}$ & $\begin{array}{l}\text { Prefer surgery/consider surgery } \\
\text { if progression during } \\
\text { neoadjuvant treatment }\end{array}$ \\
\hline 3 & $\begin{array}{l}\text { Locally Advanced } \\
\text { Cancer }\end{array}$ & $\begin{array}{l}\text { Neoadjuvant therapy } \\
\text { (On completion of NACT and responding to } \\
\text { it, patients can continue to stay on } \\
\text { chemotherapy till surgery) }\end{array}$ & $\begin{array}{l}\text { Neoadjuvant therapy } \\
\text { (On completion of NACT and responding to } \\
\text { it, patients can continue to stay on } \\
\text { chemotherapy till surgery) }\end{array}$ & $\begin{array}{l}\text { Defer surgery until progression } \\
\text { or emergency indications }\end{array}$ \\
\hline 4 & $\begin{array}{l}\text { Emergency } \\
\text { indication- } \\
\text { Absolute } \\
\text { dysphagia/ GOO }\end{array}$ & $\begin{array}{l}\text { Prefer endoscopic procedures, if fails } \\
\text { consider for surgery }\end{array}$ & & $\begin{array}{l}\text { Prefer endoscopic procedures, } \\
\text { if fails consider for surgery }\end{array}$ \\
\hline
\end{tabular}




\section{Hepato Pancreatico Biliary Cancers}

\begin{tabular}{lllll}
\hline $\begin{array}{l}\text { SL } \\
\text { No }\end{array}$ & Subtypes & $\begin{array}{l}\text { Recommendations by - ACS, } \\
\text { SSO. }\end{array}$ & $\begin{array}{l}\text { Suggested non-surgical } \\
\text { options }\end{array}$ & Suggested surgical options \\
\hline 1. & Early stage tumors & Consider surgery & $\begin{array}{c}\text { Consider surgery if resources are } \\
\text { available }\end{array}$ \\
2 & $\begin{array}{c}\text { Borderline resectable or locally } \\
\text { advanced inoperable } \\
\begin{array}{c}\text { Emergency indication- Obstructive } \\
\text { jaundice/ GOO } \\
\text { Asymptomatic PNET, GIST, high } \\
\text { risk IPMN's }\end{array}\end{array}$ & $\begin{array}{c}\text { Neoadjuvant treatment } \\
\text { Prefer endoscopic procedures, if } \\
\text { fails consider for surgery } \\
\text { Defer surgery }\end{array}$ & $\begin{array}{c}\text { Consider for neoadjuvant progression or } \\
\text { chemotherapy }\end{array}$ & $\begin{array}{c}\text { Prefer endoscopic procedures, if fails } \\
\text { consider for surgery }\end{array}$ \\
Defer surgery \\
\hline
\end{tabular}

\section{Peritoneal Surface Malignancies}

\begin{tabular}{|c|c|c|c|c|}
\hline $\begin{array}{l}\text { SL } \\
\text { No }\end{array}$ & Subtypes & $\begin{array}{l}\text { Recommendations } \\
\text { by - ACS, SSO. }\end{array}$ & Suggested non-surgical options & Suggested surgical options \\
\hline \multirow[t]{3}{*}{1.} & \multicolumn{4}{|c|}{ Pseudomyxomaperitonei and colorectal tumors } \\
\hline & $\begin{array}{l}\text { Low grade appendiceal } \\
\text { tumors }\end{array}$ & Defer Surgery & & Defer surgery \\
\hline & $\begin{array}{l}\text { High grade appendiceal } \\
\text { tumors } \\
\text { and Colorectal tumors }\end{array}$ & Defer Surgery & Neoadjuvant chemotherapy & Defer Surgery \\
\hline 2 & Mesothelioma & $\begin{array}{l}\text { Consider systemic therapy. } \\
\text { Defer Surgery }\end{array}$ & Consider systemic therapy & Defer Surgery \\
\hline 3 & Ovarian Cancer & $\begin{array}{l}\text { Consider systemic therapy. } \\
\text { Defer Surgery }\end{array}$ & $\begin{array}{l}\text { Consider systemic therapy/ } \\
\text { Metronomic chemotherapy } \\
\text { (Pazopanib/Endoxan/Etoposide) }\end{array}$ & Defer Surgery \\
\hline
\end{tabular}

\section{Thorasic Malignancies}

\begin{tabular}{|c|c|c|c|c|}
\hline $\begin{array}{l}\text { SL } \\
\text { No }\end{array}$ & Subtypes & $\begin{array}{l}\text { Recommendations by -ACS, } \\
\text { IASLC. }\end{array}$ & Suggested non-surgical options & Suggested surgical options \\
\hline 1. & $\begin{array}{l}\text { Ground glass nodules } \\
\text { Pulmonary metastases }\end{array}$ & Defer surgery & & Defer surgery \\
\hline 2 & Carcinoids & Defer surgery & & Defer surgery \\
\hline 3 & Mediastinal tumors- Thymoma & Defer surgery unless symptomatic & & Defer surgery \\
\hline 4 & $\begin{array}{l}\text { NSCLC } \\
\text { T1a/T1b and node negative }\end{array}$ & Defer surgery & $\begin{array}{l}\text { Defer surgery } \\
\text { In adenocarcinoma- consider oral targeted } \\
\text { therapy if suggested by mutational analysis }\end{array}$ & Defer surgery \\
\hline 5 & $\begin{array}{l}\text { NSCLC } \\
\text { T1c or above and } \mathrm{cN} 0, \mathrm{cN} 1\end{array}$ & $\begin{array}{l}\text { Consider early surgery for } \\
\text { operable tumors }\end{array}$ & $\begin{array}{l}\text { Neoadjuvant chemotherapy } \\
\text { In adenocarcinoma- consider oral targeted } \\
\text { therapy if suggested by mutational analysis }\end{array}$ & Defer surgery until progression \\
\hline 6 & $\begin{array}{l}\text { Emergency indication- } \\
\text { Bleeding/hemoptysis, } \\
\text { obstructed airway }\end{array}$ & $\begin{array}{l}\text { Consider non-invasive } \\
\text { intervention procedures, if fails } \\
\text { surgery }\end{array}$ & & $\begin{array}{l}\text { Consider non-invasive } \\
\text { intervention procedures, if fails } \\
\text { surgery }\end{array}$ \\
\hline
\end{tabular}




\section{Sarcomas}

\begin{tabular}{|c|c|c|c|c|}
\hline $\begin{array}{l}\text { SL } \\
\text { No }\end{array}$ & Subtypes & Recommendations by -SSO. & Suggested non-surgical options & Suggested surgical options \\
\hline 1. & $\begin{array}{l}\text { Truncal/extremity low grade } \\
\text { sarcomas (ALT, classic } \\
\text { DFSP, desmoids) }\end{array}$ & Defer surgery & & $\begin{array}{l}\text { Defer surgery and assessment } \\
\text { for progression 4-6 } \\
\text { weekly }\end{array}$ \\
\hline 2 & $\begin{array}{l}\text { High grade or recurrent } \\
\text { sarcomas }\end{array}$ & $\begin{array}{l}\text { Consider for neoadjuvant } \\
\text { treatment } \\
\text { (Chemo/radiotherapy) }\end{array}$ & NACT or NART & $\begin{array}{l}\text { Defer surgery until } \\
\text { progression }\end{array}$ \\
\hline 3 & GIST & Consider Imatinib & Consider Imatinib & $\begin{array}{l}\text { Defer surgery until } \\
\text { progression }\end{array}$ \\
\hline 4 & $\begin{array}{l}\text { Emergency indications- } \\
\text { Bleeding or obstruction }\end{array}$ & Consider for palliative surgery & $\begin{array}{l}\text { Consider palliative radiotherapy for } \\
\text { bleeding }\end{array}$ & $\begin{array}{l}\text { Consider for palliative } \\
\text { surgery }\end{array}$ \\
\hline
\end{tabular}

$N A R T$ neoadjuvant radiotherapy, NACT neoadjuvant chemotherapy

\section{Oral and Endocrine Tumors}

\begin{tabular}{|c|c|c|c|c|}
\hline $\begin{array}{l}\text { SL } \\
\text { No }\end{array}$ & Subtypes & $\begin{array}{l}\text { Recommendations by -SSO, AHNS, Irish } \\
\text { head and neck society. }\end{array}$ & Suggested non-surgical options & $\begin{array}{l}\text { Suggested surgical } \\
\text { options }\end{array}$ \\
\hline 1. & Thyroid cancer & Defer surgery & & Defer surgery \\
\hline 2 & Parathyroid & $\begin{array}{l}\text { Defer surgery unless life threatening } \\
\text { hypercalcemia }\end{array}$ & & $\begin{array}{l}\text { Defer surgery unless } \\
\text { life threatening } \\
\text { hypercalcemia }\end{array}$ \\
\hline 3 & Adrenal tumors & Defer surgery unless medically uncontrolled & & $\begin{array}{l}\text { Defer surgery unless } \\
\text { medically } \\
\text { uncontrolled }\end{array}$ \\
\hline \multirow[t]{2}{*}{4} & $\begin{array}{l}\text { Oral Cancers } \\
\text { Early \& Locally advanced }\end{array}$ & $\begin{array}{l}\text { Defer Surgery for T1 slow growing tumors } \\
\text { involving low risk sub sites with node } \\
\text { negative neck. }\end{array}$ & & \\
\hline & & Operable Locally Advanced Oral cancer & $\begin{array}{l}\text { Consider for neoadjuvant chemotherapy } \\
\text { /Chemoradiation/Oral metronomic } \\
\text { chemotherapy }\end{array}$ & $\begin{array}{l}\text { Defer surgery until } \\
\text { progression }\end{array}$ \\
\hline 5 & $\begin{array}{l}\text { Emergency indication- } \\
\text { Bleeding/hemoptysis, } \\
\text { obstructed airway }\end{array}$ & $\begin{array}{l}\text { Consider non-invasive intervention } \\
\text { procedures, if fails surgery }\end{array}$ & $\begin{array}{l}\text { Consider non surgical options (RT/ } \\
\text { embolization) }\end{array}$ & $\begin{array}{l}\text { Surgery if non surgical } \\
\text { options fail }\end{array}$ \\
\hline
\end{tabular}




\section{Gynaecologic Cancers}

\section{SL Subtypes}

No

Ovarian Cancer

2 Endometrium $\mathrm{Ca}$

(high grade/high risk uterine)

3 Early stage, low grade endometrial cancers

4 Cervical cancer

5 Emergency indications - Bleeding, bowel perforation, peritonitis, torsion, rupture of suspected malignant pelvic masses

Recommendations by - ACS, Suggested non-surgical options British gynecological cancer society.

\section{Consider systemic therapy. \\ (Defer Surgery except pelvic confined suspected masses of ovarian cancer) \\ Consider for surgery within \\ 4 weeks based on the urgency of symptoms \\ Defer surgery for 10-12 weeks}

Defer surgery for CIN

Consider surgery for early stage operable cancer

Locally advanced cancers

Surgery
Suggested surgical options

Defer Surgery

Consider systemic therapy

Metronomic chemotherapy

(Pazopanib/Endoxan/Etoposide)

Consider for alternative treatment Radiotherapy/chemotherapy/Hormo- surgery nal therapy

Defer surgery/ Consider surgery if progression

Palliative radiotherapy for bleeding

Consider surgery for early stage cancer

Consider CT+ RT

$\mathrm{CT}+\mathrm{RT}$

Surgery

\section{MELANOMA}

\begin{tabular}{|c|c|c|c|c|}
\hline $\begin{array}{l}\text { SL } \\
\text { No }\end{array}$ & Subtypes & Recommendations by (NCCN, SSO) & $\begin{array}{l}\text { Suggested non- } \\
\text { surgical options }\end{array}$ & Suggested surgical options \\
\hline 1. & Melanoma in situ & Defer surgery for upto 3 months & & $\begin{array}{l}\text { Defer surgery and assessment for } \\
\text { progression 4-6 weekly }\end{array}$ \\
\hline 2 & T1 melanomas $(\leq 1 \mathrm{~mm})$ & $\begin{array}{l}\text { Defer surgery for upto } 3 \text { months even if } \\
\text { positive margin on biopsy }\end{array}$ & & $\begin{array}{l}\text { Defer surgery and assessment for } \\
\text { progression 4-6 weekly }\end{array}$ \\
\hline 3 & Melanomas $>2 \mathrm{~mm}$ thick (T3/T4) & $\begin{array}{l}\text { Surgery should take priority over } \leq 2 \mathrm{~mm} \\
\text { (T1/T2) }\end{array}$ & & $\begin{array}{l}\text { Surgery should take priority over } \\
\leq 2 \mathrm{~mm}(\mathrm{~T} 1 / \mathrm{T} 2)\end{array}$ \\
\hline 4 & $\begin{array}{l}\text { Stage III disease (Clinically } \\
\text { palpable regional nodes) }\end{array}$ & $\begin{array}{l}\text { Defer lymphadenectomy and offer } \\
\text { neoadjuvant systemic therapy immune } \\
\text { blockade or BRAF/MEK inhibitors } \\
\text { (Exception-if node encroaching vital } \\
\text { structures eg., carotid artery, skull base). } \\
\text { Surgery should be performed } 8-9 \text { weeks } \\
\text { after initiation of neoadjuvant therapy }\end{array}$ & $\begin{array}{l}\text { Neoadjuvant } \\
\text { systemic therapy }\end{array}$ & Defer surgery \\
\hline 5 & $\begin{array}{l}\text { Metastatic resections } \\
\quad(\text { Stages III and IV) }\end{array}$ & $\begin{array}{l}\text { Defer surgery and continue systemic } \\
\text { therapy (as per hospital resources) }\end{array}$ & $\begin{array}{l}\text { Continue systemic } \\
\text { therapy }\end{array}$ & Defer surgery \\
\hline
\end{tabular}




\section{Compliance with Ethical Standards}

Conflict of Interest The authors declare that they have no conflict of interest.

\section{References}

1. Zhu N, Zhang D, Wang W, Li X, Yang B, Song J et al (2020) A Novel Coronavirus from Patients with Pneumonia in China, 2019. N Engl J Med 382(8):727-733

2. Wang D, Hu B, Hu C, Zhu F, Liu X, Zhang J et al (2020) Clinical characteristics of 138 hospitalized patients with 2019 Novel Coronavirus-infected pneumonia in Wuhan, China. JAMA 323(11):1061-1069 https://doi.org/10.1001/jama.2020.1585

3. Novel Coronavirus (2019-nCoV) situation reports [Internet]. [cited 2020 Apr 8]. Available from: https://www.who.int/emergencies/ diseases/novel-coronavirus-2019/situation-reports

4. Holshue ML, DeBolt C, Lindquist S, Lofy KH, Wiesman J, Bruce $\mathrm{H}$ et al (2020) First case of 2019 Novel Coronavirus in the United States. N Engl J Med 382(10):929-936

5. Silverstein WK, Stroud L, Cleghorn GE, Leis JA (2020) First imported case of 2019 novel coronavirus in Canada, presenting as mild pneumonia. Lancet 395(10225):734

6. Early Epidemiological and Clinical Characteristics of 28 Cases of Coronavirus Disease in South Korea. Osong Public Health Res Perspect [Internet]. 2020 Feb [cited 2020 Apr 8];11(1):814.Available from: https://www.ncbi.nlm.nih.gov/pmc/articles/ PMC7045878/

7. March 24 O, 2020. COVID-19 Guidelines for Triage of Cancer Surgery Patients [Internet]. American College of Surgeons. [cited 2020 Apr 9]. Available from: https://www.facs.org/covid-19/ clinical-guidance/elective-case/cancer-surgery

8. COVID-19 Resources [Internet]. Society of Surgical Oncology. [cited 2020 Apr 9]. Available from: https:/www.surgonc.org/ resources/covid-19-resources/
9. NCCN covid-19 resources [Internet]. [cited 2020 Apr 9]. Available from: https://www.ncen.org/covid-19/

10. theihns. The Irish Head and Neck Society [Internet]. theihns. [cited 2020 Apr 9]. Available from: https://theihns.com/covid19

11. BASO - The British Association of Surgical Oncology :: ESSO [Internet]. [cited 2020 Apr 9]. Available from: https://www. essoweb.org/baso/

12. Guidelines and Recent Publications [Internet]. British Gynaecological Cancer Society. [cited 2020 Apr 9]. Available from: https://www.bgcs.org.uk/professionals/guidelines-forrecent-publications/

13. Liang W, Guan W, Chen R, Wang W, Li J, Xu K, Li C, Ai Q, Lu W, Liang H, Li S, He J (2020) Cancer patients in SARS-CoV-2 infection: a nationwide analysis in China. Lancet Oncol 21(3):335-337

14. Zhang L, Zhu F, Xie L, Wang C, Wang J, Chen R et al (2020) Clinical characteristics of COVID-19-infected cancer patients: a retrospective case study in three hospitals within Wuhan, China. Ann Oncol 7534(20):36383-3. https://doi.org/10.1016/j.annonc. 2020.03.296

15. Al-Shamsi HO, Alhazzani W, Alhuraiji A, Coomes EA, Chemaly RF, Almuhanna M et al (2020) A practical approach to the management of cancer patients during the novel coronavirus disease 2019 (COVID-19) pandemic: an international collaborative group. Oncologist

16. CDC. Coronavirus Disease 2019 (COVID-19)-HCP/PPE-strategy [Internet]. Centers for Disease Control and Prevention. 2020 [cited 2020 Apr 12]. Available from: https://www.cdc.gov/coronavirus/ 2019-ncov/hcp/ppe-strategy/index.html

17. WHO/Coronavirus-2019/Technical guidance/Health Workers [Internet]. [cited 2020 Apr 12]. Available from: https://www.who. int/emergencies/diseases/novel-coronavirus-2019/technicalguidance/health-workers

Publisher's Note Springer Nature remains neutral with regard to jurisdictional claims in published maps and institutional affiliations. 